\title{
Activation of the phosphorylation of ATM contributes to radioresistance of glioma stem cells
}

\author{
WEI ZHOU ${ }^{1}$, MAO SUN ${ }^{2}$, GUANG-HUI LI ${ }^{3}$, YONG-ZHONG WU ${ }^{4}$, YING WANG $^{4}$, \\ FU JIN ${ }^{4}$, YUN-YUN ZHANG ${ }^{4}$, LI YANG ${ }^{5}$ and DONG-LIN WANG ${ }^{6}$ \\ ${ }^{1}$ Chongqing Cancer Institute, Chongqing 400030; ${ }^{2}$ Department of Gastroenterology, The Second Affiliated Hospital \\ of Chongqing Medical University, Chongqing 400010; ${ }^{3}$ Department of Oncology, Institute for Cancer Research \\ of the People's Liberation Army, Xinqiao Hospital, Third Military Medical University, Chongqing 400037; ${ }^{4}$ Department \\ of Radiotherapy, Chongqing Cancer Institute, Chongqing 400030; ${ }^{5}$ School of Bioengineering, Chongqing University, \\ Chongqing 400044; ${ }^{6}$ Department of Oncology, Chongqing Cancer Institute, Chongqing 400030, P.R. China
}

Received March 15, 2013; Accepted June 7, 2013

DOI: 10.3892/or.2013.2614

\begin{abstract}
Ionizing radiation (IR) is currently the most efficient therapy available for malignant glioma. Unfortunately, this strategy is palliative due to the characteristics of radioresistance of malignant glioma. The aim of our study was to compare glioma stem cells (GSCs) with glioma cells (GCs) to determine whether GSCs are responsible for the radioresistance phenotype and to elucidate whether cell cycle checkpoint proteins are responsible for the radioresistance of GSCs. In this study, CD133 (a marker of brain cancer stem cells) and nestin were co-expressed in GSCs isolated from GCs. The percent of CD133+ cells in GSCs and GCs were $>80$ and $<2 \%$, respectively. Significantly more GSCs survived following 2, 4, 6 and 8 Gy IR than GCs. IR kills cancer cells primarily through DNA double-strand breaks (DSBs). The neutral comet assay is often used to intuitively show the level of DSBs. Significantly fewer GSCs showed DNA damage than GCs following 2 Gy IR. This demonstrated that GSCs are more resistant to in vitro radiation than GCs. Furthermore, activated ataxia telangiectasia mutated (ATM) is essential for the activation of downstream effector kinases, such as checkpoint kinase 2 (Chk2) and p53 which mainly contribute to the proper regulation of IR-induced arrest in the G1 phase. DNA damage induced by IR potently initiated activation of phosphorylation of the ATM, p53 and Chk2 checkpoint proteins. Activation of the phosphorylation of these checkpoint proteins was significantly higher in the GSCs compared to GCs. We found that inhibition of ATM activation induced cell cycle checkpoint defects and increased the rate of apoptosis of GSCs following IR. Our results suggest
\end{abstract}

Correspondence to: Professor Dong-Lin Wang, Department of Oncology, Chongqing Cancer Institute, Chongqing 400030, P.R. China E-mail: 1052308491@qq.com; donglinw@21cn.com

Key words: glioma, glioma stem cells, ionizing radiation, radiosensitivity, radioresistance that GSCs were more resistant to radiation compared to GCs due to high expression of phosphorylated cell cycle checkpoint proteins, and inhibition of ATM could significantly reduce the radioresistance of GSCs and GCs. ATM may represent a source of radioresistance in GSCs and a target of improved radiosensitivity of GSCs.

\section{Introduction}

Malignant glioma has emerged as one of the most common and deadly neurosurgical disorders in adults. Although the etiology of gliomas remains largely unknown, hereditary disorders of the central nervous system (CNS), such as neurofibromatoses and the tuberous sclerosis complex, have been implicated as predisposing risk factors (1). The 5-year survival rate of malignant glioma is less than $15 \%$ and most patients die within 18 months following diagnosis (2), making this form of cancer particularly devastating. The most common treatment strategy involves surgical resection followed by radiotherapy. Surgical resection of glioma is usually incomplete. Therefore, the residual or metastasized cancer cells often necessitate subsequent radiotherapy. Although radiotherapy can prolong the length of survival, its effect is generally modest. Recent technical advances in radiotherapy, such as intensity-modulated radiation therapy that avoids non-cancerous tissue, have only marginally improved its clinical efficacy (3-5). However, malignant gliomas are frequently resistant to radiation therapy, which not only complicates treatment but also impairs the survivor's quality of life.

Although the exact mechanism underlying glioma radioresistance has remained elusive, several studies have implicated glioma stem cells (GSCs) as the potential source of this characteristic. In general, tumor stem-like cells (TSCs) have become a recent focus of research aiming to understand treatment response, and TSCs have been shown to play major roles in determining sensitivity or resistance to chemotherapy and radiotherapy. Bao et al (6) characterized the subset of glioma cells (GCs) that survived ionizing radiation (IR) and determined that these tumor cells expressed the CD133 stem cell 
marker; in addition, the authors showed that the $\mathrm{CD} 133^{+}$glioma cells were able to repair radiation-induced DNA damage more effectively than the CD133- glioma cells and underwent less apoptosis following irradiation. However, a subsequent study by McCord et al (7) demonstrated that a portion of CD133+ ${ }^{+} \mathrm{TSCs}$ could more sensitively respond to radiation and showed that this radiosensitive subset of cells had a different DNA damage response than the cells of the traditional glioblastoma in vitro model. These results suggest that $\mathrm{CD} 133^{+}$cells may represent an in vitro model system useful for studying the detailed mechanisms that mediate the glioblastoma radioresponse. To identify the radiosensitivity of $\mathrm{CD} 133^{+} \mathrm{GSCs}$, it is necessary to further compare the radiosensitivity of CD133+ GSCs with established cell lines by clonogenic analysis and comet assay.

Cell cycle checkpoint responses play essential roles in DNA strand breaks (DSBs) repair by IR and thus induce cell cycle arrest to repair damaged DNA. It is well-established that ataxia telangiectasia mutated (ATM) protein is critical for initiation of these checkpoint pathways (8). ATM kinase and its downstream substrates, p53 and checkpoint kinase 2 (Chk2), have been implicated in the control of the $\mathrm{G} 1$ phase checkpoint pathways (8-12). However, the roles of ATM and its substrates in the radiosensitivity of GSCs are not clear. To observe the effects of ATM in the radiosensitivity of GSCs, KU55933 as a specific and reversible inhibitor of ATM, was used in our study. We found that ATM played an important role in the radioresistance of GSCs.

\section{Materials and methods}

Cell culture. Two glioma cell lines were used in this study: U87 and U251. The glioma cell lines were grown in monolayer in Dulbecco's modified Eagle's medium (DMEM) (Invitrogen Life Technologies, Carlsbad, CA, USA) with $10 \%$ fetal bovine serum (FBS) (HyClone, Logan, UT, USA). The glioma cell lines were separated into $\mathrm{CD}_{133^{+}}$and $\mathrm{CD} 133^{-}$cells according to the CD133/2-PE antibody (Miltenyi Biotec) as a surface marker by fluorescence-activated cell sorting (FACS). Isolated $\mathrm{CD}_{133^{+}}$cells were then cultured in neural stem cell medium (NSC-M) supplemented with $20 \mathrm{ng} / \mathrm{ml}$ of basic fibroblast growth factor (bFGF), $20 \mathrm{ng} / \mathrm{ml}$ epidermal growth factor (EGF) (both from Peprotech, Inc., Rocky Hill, NJ, USA), 10 ng/ml leukemia inhibitory factor (LIF) (Chemicon, Temecula, CA, USA) and a 1:50 dilution of B27 (Gibco-BRL, Carlsbad, CA, USA). The cells were grown at $37^{\circ} \mathrm{C}$ in a humidified incubator with $5 \% \mathrm{CO}_{2}$ in air for $\sim 7$ days. The formed tumor spheres were harvested and used for culture and the indicated assays.

Detection of CD133 and nestin expression by immunofluorescence. Sterile coverslips were installed into 24 -well plates. GSCs were collected by centrifuging at $1,000 \mathrm{rpm}$. Then, single-cell suspensions were plated at a $1 \times 10^{5} / \mathrm{ml}$ cell density. Medium was supplemented with NSC-M in order to observe expression of CD133 and nestin. After culturing in NSC-M for 7-8 days, the tumor spheres were formed, washed 3 times with phosphate-buffered solution (PBS; 5 min each), and fixed by incubating with $4 \%$ polyoxymethylene (for $30 \mathrm{~min}$ ). After an additional 3 PBS washes (5 min each), $0.5 \%$ Triton X-100 was added and the sample was then immunoreacted with $10 \%$ FBS and $50 \mu \mathrm{l}$ of CD133 and nestin antibodies (1:50; Zhongshan
Biotechnology, China) by incubating at $4^{\circ} \mathrm{C}$ for $12 \mathrm{~h}$. After an additional 3 PBS washes (5 min each), the TRITC and FITC secondary antibodies (1:50; Zhongshan Biotechnology) were added and immunoreacted by incubating at $37^{\circ} \mathrm{C}$ for $1 \mathrm{~h}$. After a final 3 PBS washes (5 min each), DAPI (Beyotime Institute of Biotechnology) staining was added and the immunoreactivity was observed with a Leica rapid scanning laser confocal microscope.

Detection of the percentage of CD133 cells by flow cytometry $(F C M)$. A total of $1 \times 10^{5} \mathrm{GCs}$ and GSCs was collected by centrifuging at $1,000 \mathrm{rpm}$. Then, the cells were mixed with $20 \mu \mathrm{l}$ of FcR blocking reagent, $10 \mu \mathrm{l}$ of CD133/1-PE antibody, or $10 \mu \mathrm{l}$ of mouse IgG2b (all from Miltenyi Biotec). The mixture was incubated at $4^{\circ} \mathrm{C}$ for $10 \mathrm{~min}$. After adding $800 \mu \mathrm{l}$ of PBS, the treated cells were collected by centrifuging at $1,000 \mathrm{rpm}$ and washed with $500 \mu \mathrm{l}$ PBS. The percentage of $\mathrm{CD}_{133}{ }^{+}$cells in the sample was determined by FCM analysis (Beckon-Dickinson, Franklin Lakes, NJ, USA).

Detection of multipotency by immunofluorescence. Sterile coverslips were installed into 24 -well plates. GSCs were collected by centrifuging at $1,000 \mathrm{rpm}$. Then, single-cell suspensions were plated at a $1 \times 10^{4} / \mathrm{ml}$ cell density. Medium was supplemented with $10 \%$ FBS in order to observe the differentiation of GSCs. After 7-8 days, glial fibrillary acidic protein (GFAP), mitogen-activated protein (MAP2) and myelin basic protein (MBP) were examined by immunofluorescence. The examining method was the same as for the detection of CD133 and nestin. All antibodies of GFAP, MAP2 and MBP (1:50) were provided by a commercial company (Zhongshan Biotechnology).

Detection of tumorigenicity by transplantation of GSCs and GCs in vivo. Twelve Balb/C nude mice (4 weeks old; The Experimental Animal Laboratories, Shanghai) were anesthetized with i.p. ketamine and xylazine, and GCs and GSCs of $1 \times 10^{3}, 1 \times 10^{4}$ and $1 \times 10^{5}$ were respectively implanted into the mice into the left (GCs) and right (GSCs) subcutaneous regions of the back, near lower extremity. Tumor growth was monitored weekly. The mice were sacrificed at the 8th week after implantation. The same experiment was repeated once with identical conditions. All the animal experiments were performed in strict accordance with the Institutional Animal Care guidelines.

Irradiation. The established cell lines and the $\mathrm{CD}_{133^{+}}$subset of cells were irradiated using a 6-MV X Rad source (SN4474; Varian) to deliver doses of 2, 4, 6 and $8 \mathrm{~Gy}$. The rate of the dose was $300 \mathrm{cGy} / \mathrm{min}$. The cells were harvested $4 \mathrm{~h}$ after irradiation.

Clonogenic survival assay. Cell survival was measured using a standard colony-forming efficiency assay. Briefly, GSCs were irradiated by doses of 2, 4, 6 and 8 Gy X-rays, respectively and then disaggregated into a single-cell suspension and diluted to a final concentration of $1 \times 10^{3}$ cells $/ \mathrm{ml}$. A 2-ml cell suspension was added to each well of 6 -well culture plates. The plates were incubated at $37^{\circ} \mathrm{C}$ in $5 \% \mathrm{CO}_{2}$ for 2 weeks. Prior to colony counting, cells were washed (PBS), fixed [0.0037\% (v/v) form- 
aldehyde], and stained $(0.1 \% \mathrm{w} / \mathrm{v}$-crystal violet $)$, rinsed $\left(\mathrm{dH}_{2} \mathrm{O}\right)$ and dried. Colonies consisting of $>50$ cells were counted as 1 surviving colony. Data were calculated as the surviving fraction relative to the control plates. Survival assays were repeated at least 3 times.

Single-cell gel electrophoresis (neutral comet assay). The single-cell gel electrophoresis assay was carried out with the Comet Assay kit (Trevigen), according to the manufacturer's instructions. Briefly, $4 \mathrm{~h}$ after monolayer cultures were irradiated by 2 Gy X-rays, single-cell suspensions of GCs and GSCs were generated, washed with PBS, and mixed with low melting agarose $(1: 10)$. The cell-agarose mixtures were pipetted onto the comet assay slides. Cell lysis was carried out by incubating at $4^{\circ} \mathrm{C}$ for $2.5-3 \mathrm{~h}$, after which the cells were subjected to electrophoresis for $20-25 \mathrm{~min}$ at $4^{\circ} \mathrm{C}$. The resolved samples were fixed and the DNA was visualized by staining with $5 \mu \mathrm{g} / \mathrm{ml}$ Goldview (SBS Genetech) and observation with a confocal laser microscope. Digital fluorescent images were obtained and used to calculate the percentage of comet tails/100 cells.

Western blotting. Cells were collected on dry ice, and $1 \mathrm{ml}$ total protein extraction kit containing protease inhibitor was added. Total protein was extracted after homogenization. Coomassie brilliant blue staining was used to examine the protein concentration. Subsequently, SDS-PAGE (10\% gel) electrophoresis was used to separate the proteins (50 $\mathrm{mg}$ of protein/sample), and proteins were transferred to a polyvinylidene fluoride (PVDF) membrane. The membrane was then incubated overnight with antibodies diluted to $1: 1,000$ (5\% w/v-BSA/ TBST), followed by secondary antibodies diluted to $1: 1,000$ (Zhongshan Biotechnology), incubated, and then subjected to film development and further analysis. Antibodies included: ATM and phos-ATM (S1981) (Epitomics); p53, phos-p53 (S15), Chk2 and phos-Chk2 (T68) (Santa Cruz Biotechnology Inc., Santa Cruz, CA, USA).

Drug treatment. To observe the effects of ATM on the radiosensitivity of GSCs and GCs, KU55933 was added as an ATM specific inhibitor into the culture solution at a concentration of $10 \mu \mathrm{M} 4 \mathrm{~h}$ prior to IR. Then, the cells were irradiated by 2 Gy X-rays and $4 \mathrm{~h}$ later were harvested followed by cell cycle phase and cell apoptosis analyses by FCM.

Cell cycle phase and cell apoptosis analyses by FCM. The distribution of cells in the various phases of the cell cycle was determined by FCM. Single-cell suspensions of GCs and GSCs $\left(1 \times 10^{5}\right.$ cells $\left./ \mathrm{ml}\right)$ were washed twice with PBS and fixed with $75 \%$ alcohol. After treatment with $500 \mu \mathrm{l}$ of $1 \mathrm{~g} / \mathrm{l}$ RNase at $37^{\circ} \mathrm{C}$ for $30 \mathrm{~min}$, the cells were collected for fixation again, stained with propidium iodide (PI), and analyzed by FCM. Single-cell suspensions of GCs and GSCs $\left(1 \times 10^{5}\right.$ cells $\left./ \mathrm{ml}\right)$ were aliquoted $(100 \mu \mathrm{l})$ into microcentrifuge tubes and mixed with $5 \mu \mathrm{l}$ of Annexin IV-FITC and $20 \mu \mathrm{g} / \mathrm{ml}$ PI. After $15 \mathrm{~min}$ of incubation at room temperature, the percentage of apoptotic cells were analyzed by FCM.

Statistical analysis. All statistical analyses were carried out using the SPSS software package (version 17.0 SPSS). Data are expressed as the means \pm standard deviation (SD). The significance of intergroup differences was analyzed by the t-test, followed by the Student-Newman-Keuls test for multiple comparisons. A p-value of $<0.05$ was considered statistically significant.

\section{Results}

Identification of GSCs: nestin and CD133 expression. After $24 \mathrm{~h}$ of culturing in NSC medium, isolated CD133 ${ }^{+}$cells formed neurospheres composed of 3-5 cells. Over the next 7 to 8 days, the neurospheres increased in size, reaching an average of $200 \mu \mathrm{m}$ (range, 100-300 $\mu \mathrm{m}$ ) (Fig. 1A). CD133 and nestin expression was detected in the neurospheres (Fig. 1B). The percentage of $\mathrm{CD} 133^{+}$cells was significantly higher in the GSCs than in the GCs $(83.03$ vs. $1.4 \%$ and 84.18 vs. $1.29 \%$ ) (Fig. 1C).

Identification of GSCs: multipotency. The pinning phenomenon of GSC neurospheres was observed $5 \mathrm{~h}$ after exposure to the $10 \%$ FBS. By $24 \mathrm{~h}$, the pod had protruded outside of the neurosphere, and by $72 \mathrm{~h}$, the neurospheres had flattened. At day 7 of culture, the bottom of the culture container was covered with GSCs. Most of the GSCs expressed the astrocyte-specific GFAP marker, but only a small proportion of GSCs expressed the neuron-specific MAP2 and the oligocyte-specific MBP markers (Fig. 2).

Identification of GSCs: tumorigenicity. Our study showed that transplanted tumors formed in vivo after implanting 1,000-100,000 GSCs subcutaneously into nude mice, while at least $100,000 \mathrm{GCs}$ were implanting to form transplanted tumors in vivo. The transplanted glioblastoma was characterized by H\&E staining and immunohistochemistry for GFAP in the transplanted tumor tissue sections. These results indicated that GSCs more easily formed tumors than GCs (Fig. 3).

Clonogenic survival assay after irradiation. To compare the intrinsic radiosensitivities of GSCs and established GC lines, it was necessary to perform clonogenic analyses under optimal growth conditions for GSCs and GCs. The surviving fraction of GSCs was higher than that of GCs at doses of radiation such as 2, 4, 6 and 8 Gy (p<0.05) (Fig. 4A).

Single-cell gel electrophoresis (neutral comet assay). Before irradiation, none of the GSCs or GCs formed comet tails in the single-cell gel electrophoresis. However, $4 \mathrm{~h}$ after monolayer cultures were irradiated by 2 Gy X-rays, part of the cells of both cell types showed comet tails. GCs appeared to be more sensitive to irradiation as the number of cells with comet tails was significantly greater than the number of cells with comet tails in the GSCs $(\mathrm{p}<0.05)$ (Fig. 4B and C).

Cell cycle checkpoint proteins: ATM, p53 and Chk2. The ATM signaling pathway is conserved from the human to the mouse and plays important roles in DNA damage checkpoint responses. In human GCs and GSCs, ATM kinase activity was induced by IR and there were strong increases in the phosphorylation of p53 (S15) and Chk2 (T68) relative to cells without IR (Fig. 7). In addition, activation of the phosphoryla- 


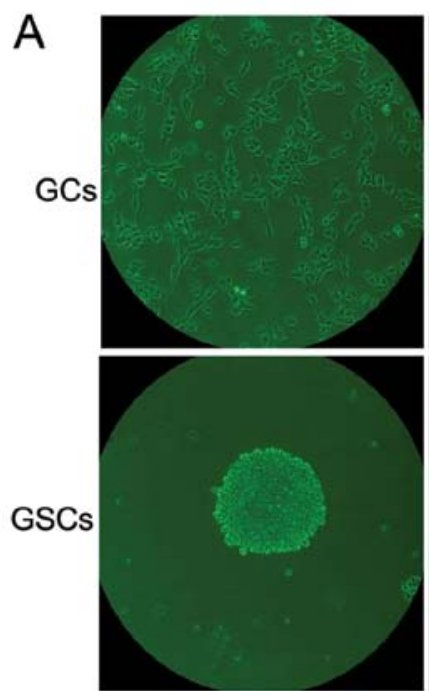

U87

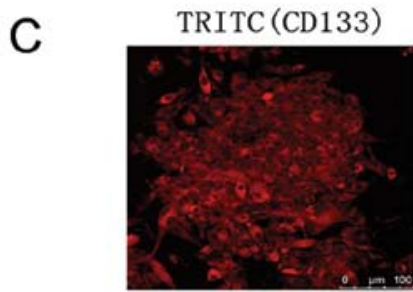

B
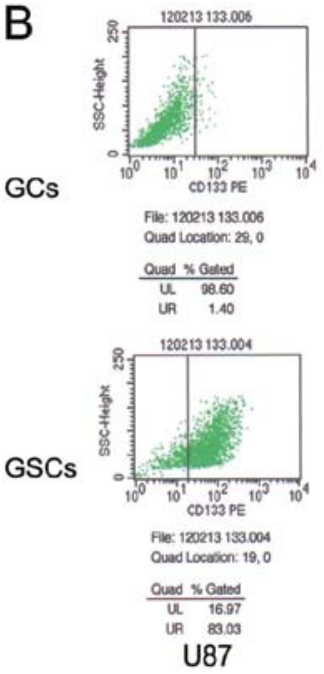

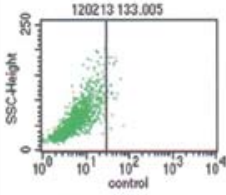

File: 120213 133.005

$\frac{\text { Ouad } 5 \text { Gated }}{u}$

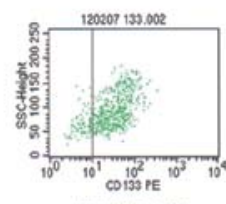

Fie: 1202007 133000

$\frac{\text { Onod } \times \text { catod }}{14}$

U251

U251

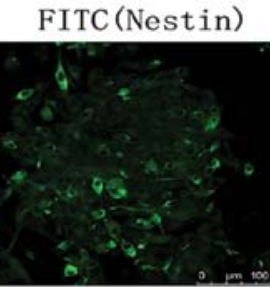

DAPI
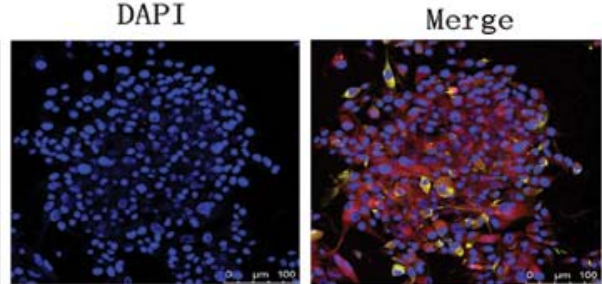

Figure 1. Identification of GSCs. (A) The glioma U87 and U251 cells formed glioma stem cell neurospheres. Magnification, x100. (B) CD133 (red) and nestin (green) expression in glioma stem cell neurospheres. Nuclei are shown in blue. Caliber, $100 \mu \mathrm{m}$. (C) Flow cytometric detection of CD133 ${ }^{+}$cells. The percentage of $\mathrm{CD}_{133^{+}}$cells in the GSCs and GCs was $>80 \%$ and $<2 \%$, respectively.
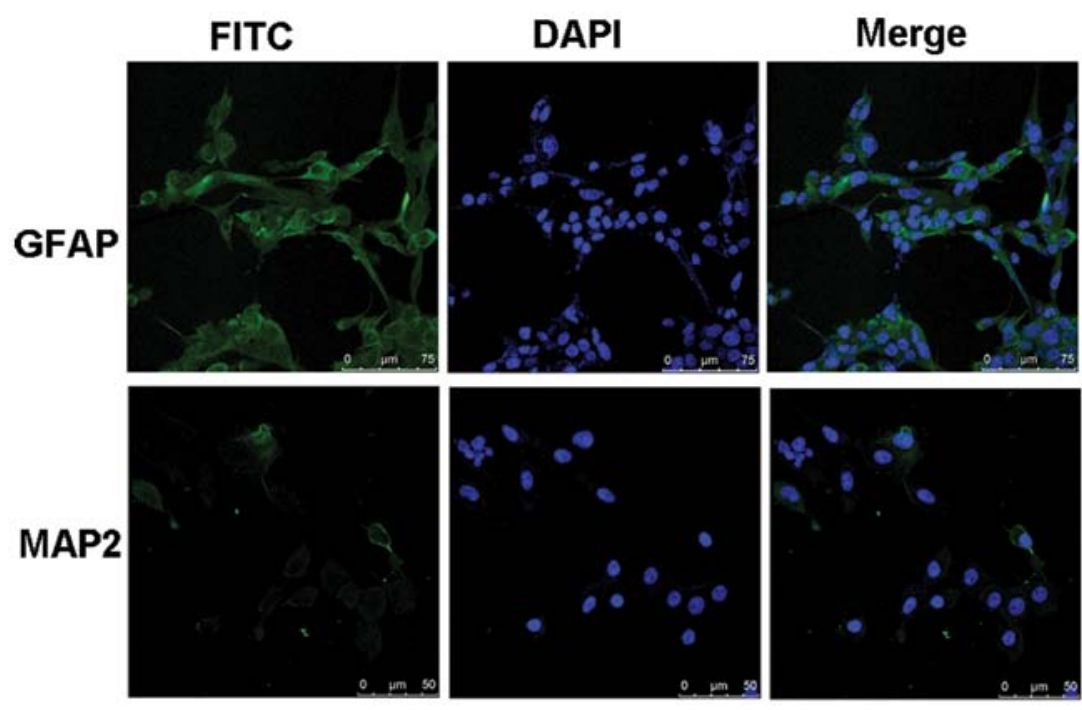

TRITC

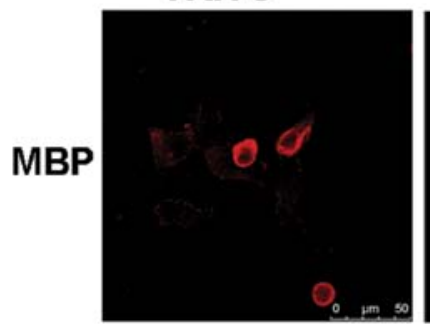

DAPI

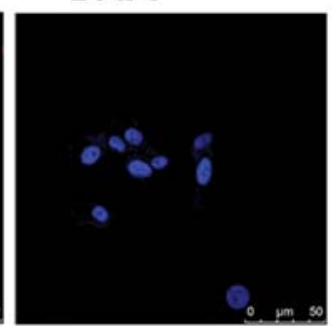

Merge

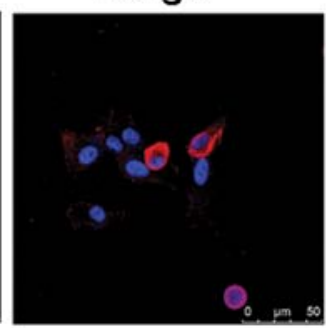

Figure 2. Multipotency of GSCs. Expression of astrocyte-specific GFAP (green) and oligo-specific MBP (red) in GSCs, as detected by immunofluorescence assay. Note that neuron-specific MAP2 (green) was observed in only a small number of cells. Nuclei are shown in blue. Caliber, $75 \mu \mathrm{m}$ (GFAP), $50 \mu \mathrm{m}$ (MAP2 and MBP). 

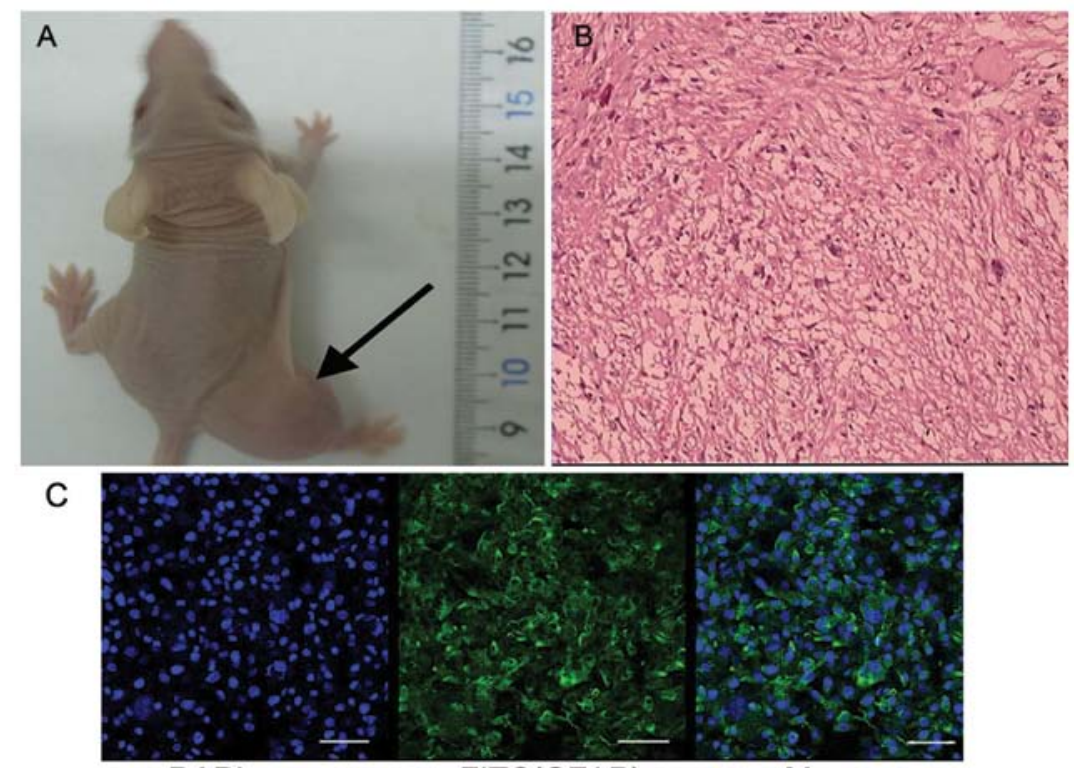

DAPI

FITC(GFAP)

Merge

Figure 3. Tumorigenicity of GSCs. (A) The transplanted tumor (arrow) formed in vivo after implantation of 1,000 GSCs subcutaneously into nude mice, while $1,000 \mathrm{GCs}$ could not form transplanted tumors in vivo. (B and C) The transplanted glioblastoma was characterized by H\&E staining and immunohistochemistry for GFAP in the transplanted tumor tissue sections.

A

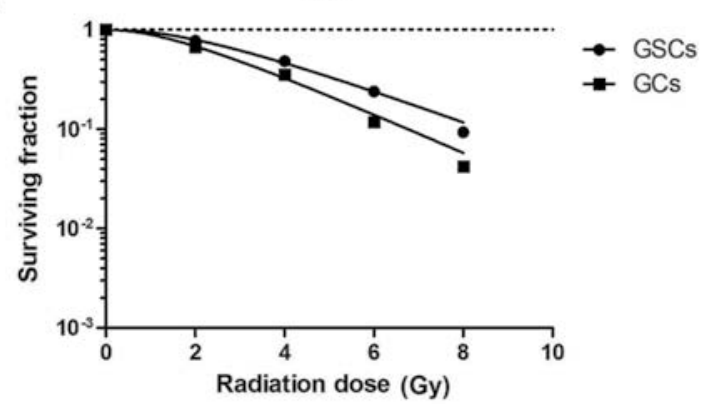

B

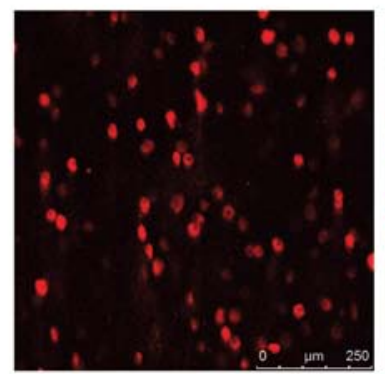

GCs (Before irradiation)

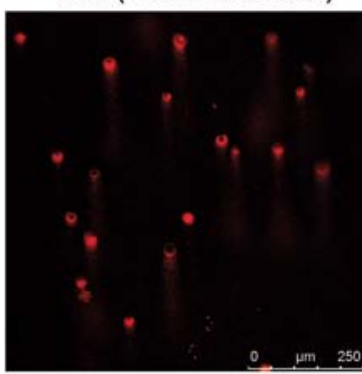

GCs (Irradiation)

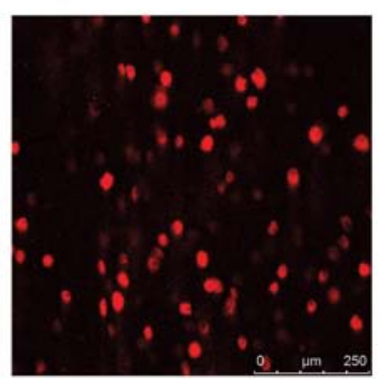

GSCs (Before irradiation)

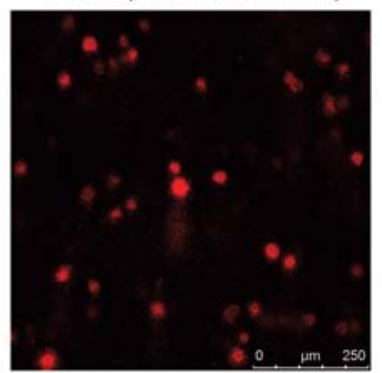

GSCs (Irradiation)
U251

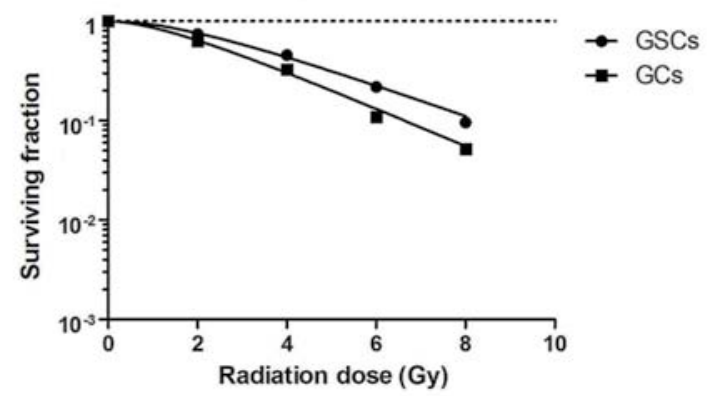

C

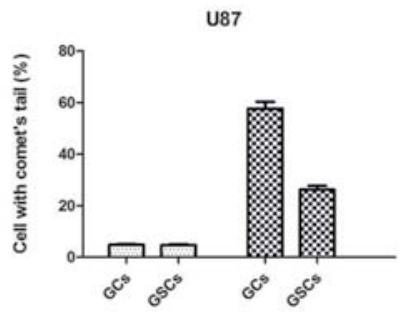

U251

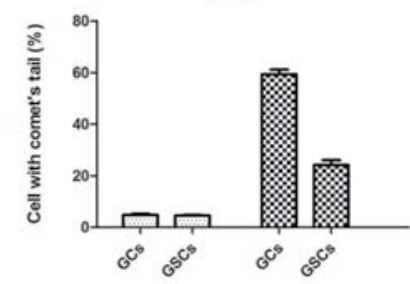

$\square$ Before irradiation Irradiation

Figure 4. Clonogenic survival assay and single-cell gel electrophoresis assay (neutral comet assay) in the GCs and GSCs. (A) The surviving fraction of GSCs was higher than that of GCs at doses of radiation of 2, 4,6 and $8 \mathrm{~Gy}$. Cell survival curve profile is representative of several repeat experiments. (B and C) Before irradiation, GCs and GSCs formed no comet tails. Comet tails were observed for both cell types. The number of cells with comet tails was significantly greater for GCs than for GSCs $4 \mathrm{~h}$ after 2 Gy irradiation $(\mathrm{p}<0.05)$. Data are the means \pm SD. Bars represent the standard error of the mean. 
A

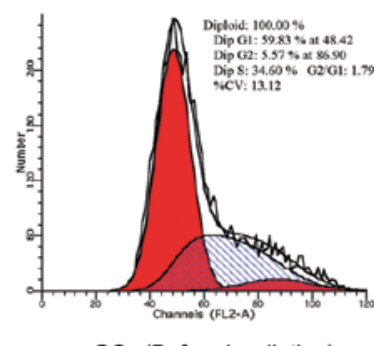

GCs (Before irradiation)

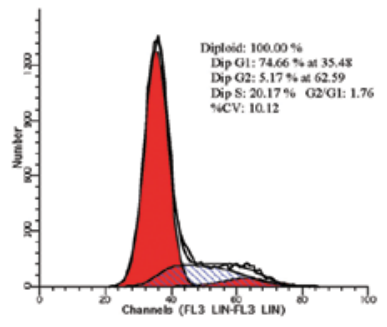

GSCs (Before irradiation)

B

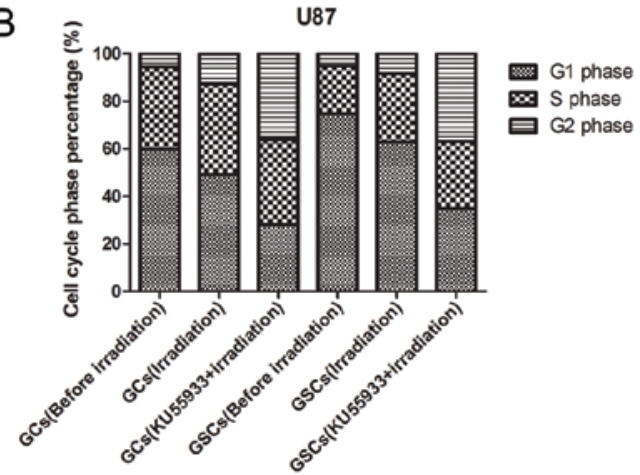

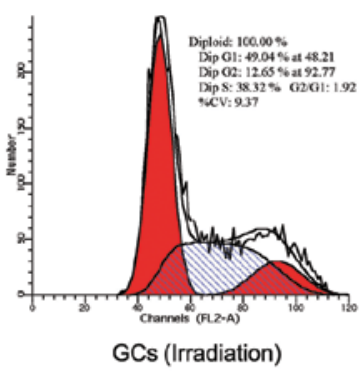

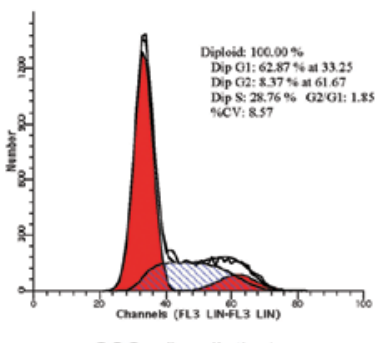

GSCs (Irradiation)

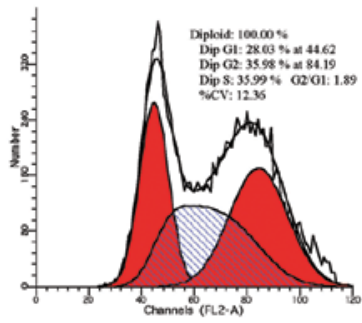

GCs (KU55933+Irradiation)

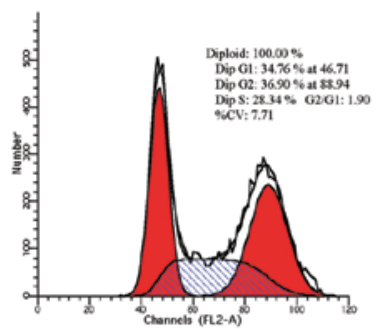

GSCs (KU55933+Irradiation)

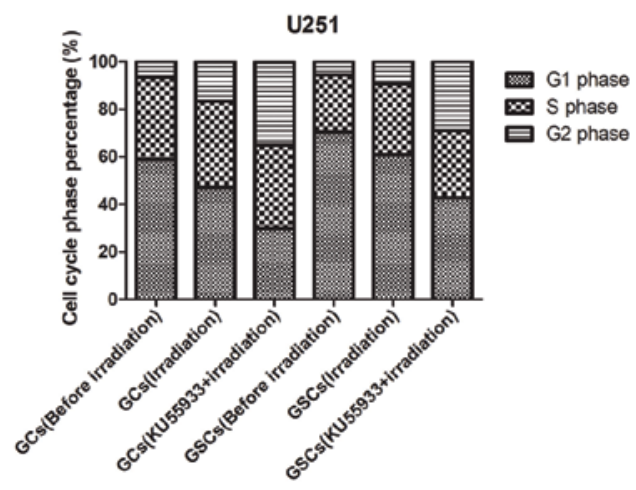

Figure 5. (A) Irradiation-induced effects on the cell cycle distribution by FCM. (B) The percentage of GCs and GSCs in the G2 phase was similar prior to irradiation. After $2 \mathrm{~Gy}$ irradiation, both cell types showed higher percentages of cells in the G2 phase ( $\mathrm{p}<0.05)$. Furthermore, the GCs responded with more cells in the G2 phase than GSCs $(\mathrm{p}<0.05)$. In response to IR, GCs and GSCs treated with KU55933 all consisted of an enhanced proportion of cells in the G2 phase and a decreased proportion of cells in the G1 phase relative to the untreated cells $(\mathrm{p}<0.05)$. Cell cycle profiles are representative of several repeat experiments.

tion of these checkpoint proteins was significantly higher in GSCs than in GCs,indicating that $\mathrm{CD} 133^{+}$cells showed more radioresistance in response to DNA damage by preferential checkpoint activation.

Irradiation-induced effects on cell cycle distribution and cell apoptosis. Prior to irradiation, there was no difference between the amount of GCs and GSCs in the G2 phase ( $>00.05)$. After 2 Gy irradiation, both cell types had a higher percentage of cells in the G2 phase $(\mathrm{p}<0.05)$. Furthermore, GCs responded with a higher percentage of cells in the G2 phase when compared with the GSCs $(\mathrm{p}<0.05)$. In response to IR, GCs and GSCs treated with KU55933 had an enhanced proportion of cells in the G2 phase and a decreased proportion of cells in the $\mathrm{G} 1$ phase relative to the untreated cells $(\mathrm{p}<0.05)$ (Fig. 5). Prior to irradiation, there was no significant difference between the percentage of cells undergoing apoptosis $(\mathrm{p}>0.05)$. After 2 Gy irradiation, both cell types consisted of a higher number of apoptotic cells, and the apoptotic rate of the GCs was significantly higher than that of the GSCs $(\mathrm{p}<0.05)$. In response to IR, GCs and GSCs treated with KU55933 all resulted in an enhanced apoptotic rate relative to the untreated cells ( $\mathrm{p}<0.05)$ (Fig. 6).

\section{Discussion}

Radiobiologists or radiation oncologists have known for many years that gliomas respond poorly to radiation therapy (13), although no experimental system has yet provided definitive evidence to establish whether this property of radioresistance is inherent or acquired. It has been shown that glioblastoma cells expressing the stem cell marker CD133 have a higher radioresistant quality than CD133- tumor cells (6). This finding has become the basis for the tumor stem cell theory of malignant glioma pathogenesis and prognosis. Subsequent studies have shown that GSCs are capable of initiating and reforming the original cancer after isolation from the primary tumor tissue (14), and to have a more robust resistance to chemotherapy and radiotherapy than non-tumorigenic cells.

CD133 is a glycoprotein that is specifically localized to cellular protrusions and has been detected in a wide range of human cancer and stem cells, including glioblastoma, hematopoietic stem cells, endothelial progenitor cells, neuronal stem cells and glial stem cells. Another marker that is often detected in neural stem cells associated with tumors is nestin, an intermediate filament protein that is expressed mostly in 
A

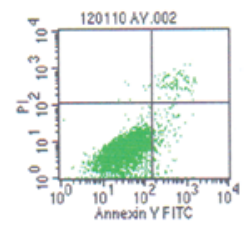

GCs (Before irradiation)

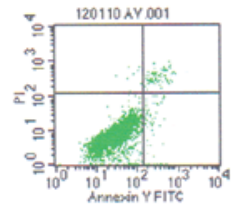

GSCs (Before irradiation)
B

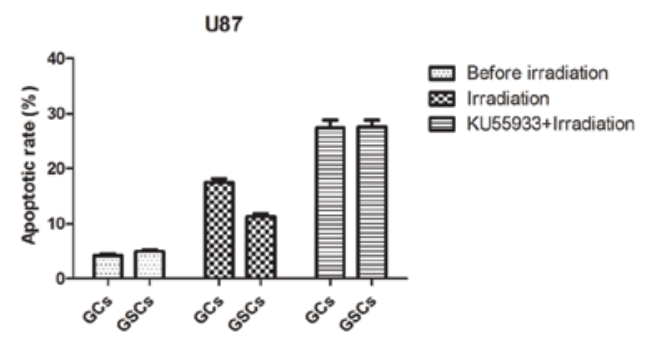

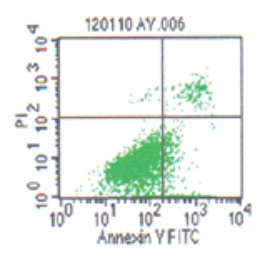

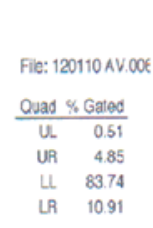

GCs (Irradiation)

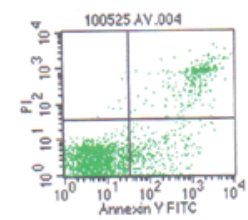

GCs (KU55933+Irradiation)

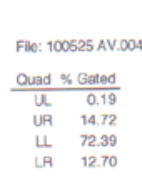

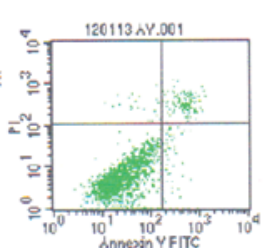

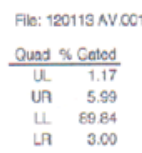

GSCs (Irradiation)
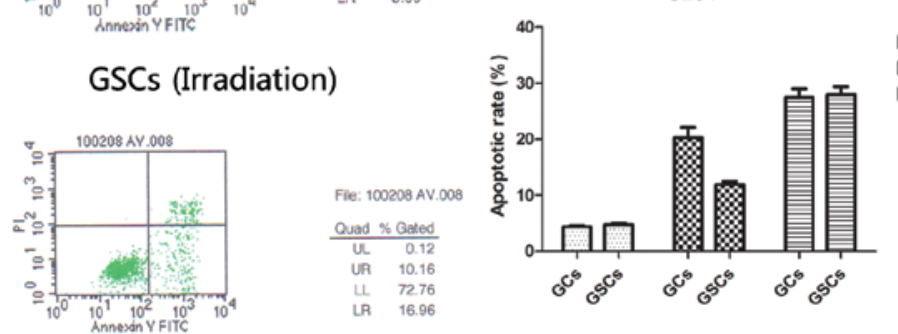

$\square$ Before irradiation $\infty$ Irradiation EU55933+Irradiation

Figure 6. (A) Irradiation-induced effects on cell apoptosis by FCM. (B) Prior to irradiation, there was no significant difference between the percent of cells undergoing apoptosis ( $>0.05$ ). After 2 Gy irradiation, both cell types had more apoptotic cells, and the apoptotic rate of the GCs was significantly higher than that of the GSCs $(\mathrm{p}<0.05)$. In response to IR, GCs and GSCs treated with KU55933 all resulted in an enhanced apoptotic rate relative to the untreated cells $(\mathrm{p}<0.05)$. FCM analysis was performed for each sample in triplicate. Data are represented as the means \pm SD. Bars represent the standard error of the mean.

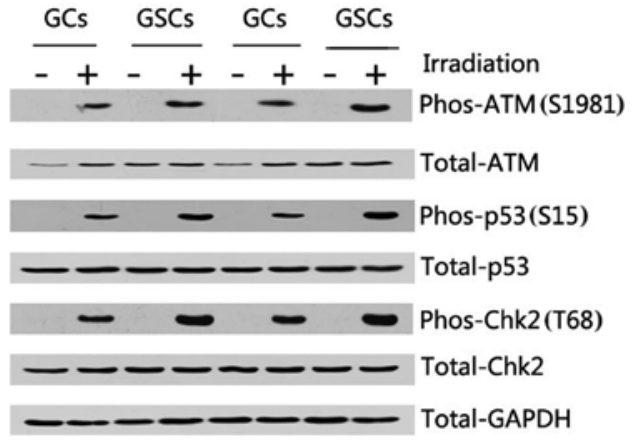

Figure 7. Expression of cell cycle checkpoint proteins ATM, p53 and Chk2 by western blotting. In GCs and GSCs, ATM kinase activity was induced by IR and there were strong increases in the phosphorylation of ATM (S1981), p53 (S15) and Chk2 (T68) relative to cells without IR (p<0.05). Furthermore, activation of the phosphorylation of these checkpoint proteins was significantly higher in GSCs than in GCs $(\mathrm{p}<0.05)$. The profiles are representative of several repeat experiments.

nerve stem cells. GSCs in our study expressed both CD133 and nestin, and at significantly higher levels than in GCs. GSC neurospheres formed secondary spheres by cell passage. This finding suggests that GSCs are capable of self-renewal $(15,16)$. GSCs can be induced to differentiate into cells expressing GFAP, MAP2 and MBP, suggesting that GSCs are multipotent and capable of differentiating into different cell types (17). Furthermore, GSCs were more capable at initiating tumors in nude mice than GCs.
Radiosensitivity is a critical determining factor of a patient's response to radiotherapy and prognosis of malignant glioma (18). In our study, we found that more GSCs than GCs survived following different doses of irradiation. Irradiation is believed to kill tumor cells by inducing irreparable DNA damage and the subsequent apoptosis mechanism. Tumor cells that are actively replicating their DNA may produce different results in gel electrophoresis than their quiescent counterparts, and this difference may be interpreted as inherent sensitivity. Therefore, we used the comet assay, which is a gel electrophoresis-based method, to measure DNA damage in tumor cells $(19,20)$. We found almost no comet tail formation in the unirradiated GCs or GSCs. However, GCs formed significantly more comet tails than GSCs after irradiation, suggesting that GSCs have a more robust trend for radioresistance.

In a previous study, McCord et al (7) demonstrated that a portion of $\mathrm{CD}_{133^{+}}$TSCs from human glioblastoma surgical specimens was more radiosensitive. In contrast, our study and the results of Bao et al (6) demonstrated that CD133 ${ }^{+}$ cells were more resistant to radiation than CD133- cells or established GCs. The discrepancy may be explained by the different radiosensitivity of different regions even in the same surgical specimen $(21,22)$. DNA damage by IR activates the DNA damage response (DDR) an intricate signal transduction pathways that involves DNA repair and activation of cell cycle checkpoints (23). Loss of function of critical proteins from DDR pathways can cause cells to have enhanced sensitivity to DNA damage (24). The ATM kinase is an important component of these DDR pathways, and cells deficient for ATM (A-T) display hypersensitivity to DSBs by IR (25). The activated 
ATM is essential for the activation of downstream effector kinases, such as Chk2 and p53, which mainly contribute to the proper regulation of IR-induced cell cycle arrest in the G1 phase (26). Transient inhibition of ATM kinase is sufficient to enhance cellular sensitivity to ionizing radiation. Our study indicates that both GSCs and GCs showed a marked increase in the phosphorylation of ATM, p53 and Chk2 checkpoint proteins in response to DNA damage relative to cells prior to IR. Furthermore, activation of the phosphorylation of these checkpoint proteins was significantly greater in GSCs than in GCs. The data presented here showed that the preferential checkpoint activation may contribute to the resistance of $\mathrm{CD}_{133^{+}}$glioma cells.

Based on these phenomena, specific inhibition of ATM function in combination with current radio-therapeutic treatment may lead to enhanced cancer cell killing. KU55933 is capable of sensitizing many types of human cancer cell lines to IR $(27,28)$. In fact, ATM and its phosphorylated downstream substrates participate in the proper regulation of IR-induced arrest in the G1, S, and G2 phases of the cell cycle. The distribution of the cell cycle in the established cell lines used in our study was mainly in the G1 phase. Fibroblasts in A-T patients showed a defective cell cycle checkpoint; p53-dependent G1 arrest did not occur (29) and therefore resulted in a prolonged G2 phase. In our study, although both GSCs and GCs untreated by KU55933 showed an increasing percentage of cells in the G2 phase in response to IR, GCs responded with more cells in the G2 phase than GSCs due to higher expression of ATM and its downstream substrates in GSCs than in GCs. However, both GSCs and GCs pretreated by KU55933 showed a significantly increased percentage of cells in the $\mathrm{G} 2$ phase in response to IR relative to untreated cells. There was no difference between GSCs and GCs when pretreated with KU55933. The results showed that inhibition of ATM led to loss of G1 phase arrest and therefore prolonged the G2 phase in GSCs and GCs; G2 phase is the most susceptible phase in the entire cell cycle in response to IR. ATM also functions in the regulation of apoptosis. Our study demonstrated that inhibition of ATM kinase induced increased apoptosis in both GSCs and GCs in response to IR.

In summary, we isolated and identified GSCs from malignant glioma cells. GSCs were capable of self-renewal and multiple differentiation and displayed a higher capability of tumor initiation in nude mice when compared with GCs. We also demonstrated that GSCs were more resistant to radiation than GCs due to high expression of phosphorylated cell cycle checkpoint proteins. Therefore, GSCs contributed to malignant glioma radioresistance and tumor repopulation. Most importantly, we found that inhibition of ATM significantly reduced the radioresistance of GSCs and GCs. Therefore therapies targeted to checkpoint proteins in preclinical and clinical development may provide an effective method by which to disrupt the resistance mechanism with the aim of improving malignant glioma control with radiation treatment.

\section{Acknowledgements}

We acknowledge grant support from the National Natural Science Foundation of China (nos. 81172387 and 30973074).

\section{References}

1. Grossman SA and Batara JF: Current management of glioblastoma multiforme. Semin Oncol 31: 635-644, 2004

2. Nieder C, Andratschke N, Wiedenmann N, Busch R, Grosu AL and Molls M: Radiotherapy for high-grade gliomas. Does altered fractionation improve the outcome? Strahlenther Onkol 180: 401-407, 2004.

3. Reya T, Morrison SJ, Clarke MF and Weissman IL: Stem cells, cancer, and cancer stem cells. Nature 414: 105-111, 2001.

4. Marx J: Cancer research. Mutant stem cells may seed cancer. Science 301: 1308-1310, 2003.

5. Clarke MF, Dick JE, Dirks PB, Eaves CJ, Jamieson $\mathrm{CH}$, Jones DL, Visvader J, Weissman IL and Wahl GM: Cancer stem cells - perspectives on current status and future directions: AACR Workshop on cancer stem cells. Cancer Res 66: 9339-9344, 2006.

6. Bao S, Wu Q, McLendon RE, Hao Y, Shi Q, Hjelmeland AB, Dewhirst MW, Bigner DD and Rich JN: Glioma stem cells promote radioresistance by preferential activation of the DNA damage response. Nature 444: 756-760, 2006.

7. McCord AM, Jamal M, Williams ES, Camphausen K and Tofilon PJ: CD133+ glioblastoma stem-like cells are radiosensitive with a defective DNA damage response compared with established cell lines. Clin Cancer Res 15: 5145-5153, 2009.

8. Tichý A, Vávrová J, Pejchal J and Rezácová M: Ataxiatelangiectasia mutated kinase (ATM) as a central regulator of radiation-induced DNA damage response. Acta Medica (Hradec Kralove) 53: 13-17, 2010.

9. Bower JJ, Zhou Y, Zhou T, Simpson DA, Arlander SJ, Paules RS, Cordeiro-Stone M and Kaufmann WK: Revised genetic requirements for the decatenation G2 checkpoint: the role of ATM. Cell Cycle 9: 1617-1628, 2010.

10. Banin S, Moyal L, Shieh S, Taya Y, Anderson CW, Chessa L, Smorodinsky NI, Prives C, Reiss Y, Shiloh Y and Ziv Y: Enhanced phosphorylation of p53 by ATM in response to DNA damage. Science 281: 1674-1677, 1998.

11. Matsuoka S, Huang M and Elledge SJ: Linkage of ATM to cell cycle regulation by the Chk2 protein kinase. Science 282: 1893-1897, 1998.

12. Jiang H, Reinhardt HC, Bartkova J, Tommiska J, Blomqvist C, Nevanlinna H, Bartek J, Yaffe MB and Hemann MT: The combined status of ATM and p53 link tumor development with therapeutic response. Genes Dev 23: 1895-1909, 2009.

13. Ignatova TN, Kukekov VG, Laywell ED, Suslov ON, Vrionis FD and Steindler DA: Human cortical glial tumors contain neural stem-like cells expressing astroglial and neuronal markers in vitro. Glia 39: 193-206, 2002.

14. Singh SK, Clarke ID, Terasaki M, Bonn VE, Hawkins C, Squire J and Dirks PB: Identification of a cancer stem cell in human brain tumors. Cancer Res 63: 5821-5828, 2003.

15. Kondo T, Setoguchi T and Taga T: Persistence of a small subpopulation of cancer stem-like cells in the C6 glioma cell line. Proc Natl Acad Sci USA 101: 781-786, 2004.

16. Li G, Chen Z, Hu YD, Wei H, Li D, Ji H and Wang DL: Autocrine factors sustain glioblastoma stem cell self-renewal. Oncol Rep 21: 419-424, 2009.

17. Li GH, Wei H, Lv SQ, Ji H and Wang DL: Knockdown of STAT3 expression by RNAi suppresses growth and induces apoptosis and differentiation in glioblastoma stem cells. Int J Oncol 37: 103-110, 2010.

18. Blazek ER, Foutch JL and Maki G: Daoy medulloblastoma cells that express CD133 are radioresistant relative to CD133- cells, and the $\mathrm{CD} 133^{+}$sector is enlarged by hypoxia. Int J Radiat Oncol Biol Phys 67: 1-5, 2007.

19. Diehn M and Clarke MF: Cancer stem cells and radiotherapy: new insights into tumor radioresistance. J Natl Cancer Inst 98: $1755-1757,2006$.

20. Singh NP, McCoy MT, Tice RR and Schneider EL: A simple technique for quantitation of low levels of DNA damage in individual cells. Exp Cell Res 175: 184-191, 1988

21. Ng WL, Yan D, Zhang X, Mo YY and Wang Y: Over-expression of miR-100 is responsible for the low-expression of ATM in the human glioma cell line: M059J. DNA Repair 9: 1170-1175, 2010.

22. Allalunis-Turner MJ, Barron GM, Day RS III, Dobler KD and Mirzayans R: Isolation of two cell lines from a human malignant glioma specimen differing in sensitivity to radiation and chemotherapeutic drugs. Radiat Res 134: 349-354, 1993. 
23. Choi S, Gamper AM, White JS and Bakkenist CJ: Inhibition of ATM kinase activity does not phenocopy ATM protein disruption: implications for the clinical utility of ATM kinase inhibitors. Cell Cycle 9: 4052-4057, 2010.

24. Kennedy RD and D'Andrea AD: DNA repair pathways in clinical practice: lessons from pediatric cancer susceptibility syndromes. J Clin Oncol 24: 3799-3808, 2006.

25. Shiloh Y: ATM and related protein kinases: safeguarding genome integrity. Nat Rev Cancer 3: 155-168, 2003.

26. Canman CE, Wolff AC, Chen CY, Fornace AJ Jr and Kastan MB: The p53-dependent G1 cell cycle checkpoint pathway and ataxiatelangiectasia. Cancer Res 54: 5054-5058, 1994.
27. Rainey MD, Charlton ME, Stanton RV and Kastan MB: Transient inhibition of ATM kinase is sufficient to enhance cellular sensitivity to ionizing radiation. Cancer Res 68: 7466-7474, 2008.

28. White JS, Choi S and Bakkenist CJ: Transient ATM kinase inhibition disrupts DNA damage-induced sister chromatid exchange. Sci Signal 3: ra44, 2010.

29. Kastan MB, Onyekwere O, Sidransky D, Vogelstein B and Craig RW: Participation of $\mathrm{p} 53$ protein in the cellular response to DNA damage. Cancer Res 51: 6304-6311, 1991. 